\title{
Robotic Tile Placement: Tools, Techniques and Feasibility
}

\author{
Nathan King $^{1 *}$, Martin Bechthold ${ }^{1}$, Anthony Kane ${ }^{1}$, and Panagiotis Michalatos ${ }^{1}$ \\ ${ }^{1}$ Harvard Graduate School of Design, Design Robotics Group \\ *Corresponding author (jking1@gsd.harvard.edu)
}

\begin{abstract}
Purpose The project develops an integrated digital workflow for robotic tile placement, a novel construction method that allows for off-site use of industrial robotics for on-site tile surfaces, and a feasibility study for implementing the approach in the context of the tile industry. The proposed approach overcomes limitations of existing methods that focus merely on efficiency. Instead, the proposition of the research is to enable the installation of unique, one-off, and highly complex tile patterns impossible to install economically by hand. Method The research uses a design experiment to develop computational and robotic technologies. Several design studies were prototyped as a proof of concept. Issues of construction integration and industry implementation were researched and advanced through interviews with experts in the field, field studies, and an extensive literature research that includes a review of U.S. tile installation standards. Results \& Discussion The novel workflow includes several computational approaches to generate tile patterns too complex to install economically by hand. Weighted randomness or patterns processed based on the analysis of bitmap images produce tile patterns within the cost-effective Rhinoceros platform. Robotic code for the running of a robotic work cell is created automatically by the integrated code generator. The code can be simulated through a newly integrated tool that permits tool path visualization as well as cost and time estimates. Regarding construction integration the research largely replaces manual tile installation to a high degree. The paper describes logistics and installation strategies for pre-tiled panels along with recommending specific material combinations. An initial feasibility study identifies fundamental parameters such as set up and placement times, adhesive application, and many more. The study allows an estimation of basic cost parameters in the context of specific construction markets, based on an assumed value of a niche tile product that employs highly complex patterns. Robotic tile placement ultimately adds value for tile producers by moving installation of specialty tile patterns to the place of tile production.
\end{abstract}

Keywords: robotics, ceramic tile, computation,

\section{INTRODUCTION}

The steady increase in the price to productivity ratio of industrial robots over the past decades is opening up new application areas that include the production of architectural ceramics. Research at Harvard University's Design Robotics Group (DRG) has been studying specific opportunities for integrating robotic technology with an initial focus on product customization and related workflow. ${ }^{1}$ The research presented in this paper summarizes a feasibility study of robotic tile placement, an area that has received little attention in recent years. The research developed a computational environment that facilitates the generation of image or pattern-based tile designs and an integrated design-to-robotic-fabrication tile placement workflow. Both are based on the low-cost and widely available digital design software platform Rhinoceros $^{T M}$ (Rhino).

The integration of robotic tile placement into the construction industry requires a re-design of current placement strategies. The envisioned system can be considered a true mass-customization approach whereby the cost of the customized product is similar to that of the standard product. The added value of complex tile pattern designs does not come at a cost premium.

Once tiles have arrived at the point of installation, the state-of-the-art, computer-controlled, highvolume tile production-to-distribution process rapidly downgrades into a purely analog handling of the material. Tile installation remains a manual process, supported by simple tools that have remained unchanged for decades. Tile installers and tile producers have few common points of contact and take little mutual interest in their related, yet sharply separate, domains. Innovation in production has not transferred to installation with its many small geographically and economically fragmented participants. Issues of tile installation warrant a new study in the context of advances in robotic technology.

In developed economies with high labor rates tile installation costs remain a major factor in the overall cost of tiled surfaces. For example, in Boston, MA, unionized tile installer labor costs for laying mosaic tile on $1 \mathrm{ft}^{2}$ netting is $30-35 \$ / \mathrm{ft}^{2}$ and non-union labor is $15-20 \$ / \mathrm{ft}^{2}$. An increasingly felt shortage of qualified construction workers especially in the United States and elsewhere has aggravated already exist- 
ing issues of installation quality. The range of feasible tile patterns is extremely limited and highly repetitive, poorly reflecting the sophisticated production technology underlying contemporary tile production.

To address these current problems of tile installation, and in an attempt to bridge the gap between producers and installers, the research investigates the use of robotic technology for tile installation as a way to address cost, time, and quality, both in terms of accuracy and design.

Existing approaches to robotic tile placement are reviewed first, followed by a report of the research conducted by Harvard's DRG. The study concludes with a discussion of issues regarding implementation and construction integration, as well as an overview of next steps needed for industry integration.

\section{Tile INSTALLATION: StATE OF THE ART}

Currently the production of complex tile patterns exists in the form of pre-assembled mosaic tile sheets, a largely automated process. While individual sheets contain complex arrangements they are used in aggregate to form repetitive patterns without the possibility of customization. The majority of custom ceramic tile mosaics are assembled by hand but several precedents do exist for the robotic placement of custom tiled mosaics. Artaic, for example, a small Boston based commercial producer of custom mosaics, uses a single robotic work-cell to fabricate custom mosaics. During the Artaic process a digital image is discretized into tile pixels using proprietary color matching software and then divided into standardized $1 \mathrm{ft}^{2}$ sheets. Each tile pixel is robotically placed into a registration grid and an adhesive sheet is applied to support the pre-assembled mosaics during shipping and installation where they are installed and grouted by hand. ${ }^{5}$

A similar, yet fundamentally different, variation is used by Top Hat Tile, which achieves image-based customization of tile mosaics through individually glazed tile pixels. In this example a $1 \mathrm{ft}^{2}$ assembly of small, unglazed tiles is loaded into a custom robotic work cell that applies or prints a custom solid colored glaze on each individual tile. Again, the robotically assembled mosaics are installed in $1 \mathrm{ft}^{2}$ sheets by hand. ${ }^{6}$

It should be mentioned that while the implementation of direct inkjet printing to create surface patterns and images, often on larger tiles, is seen in the tile industry, it is outside of the scope of this study.

\section{CONCEPT AND WORKFLOW DEVELOPMENT}

Today tile installation is done almost exclusively by hand. During this study opportunities for robotic installation were explored through two prototypical scenarios that addressed the process from computational design to physical implementation. Using integrated computational design, two modular workflows were used to generate the arrangement of tiles, as well as the machine code used to drive the robotic placement. The first workflow uses a randomized pattern of standard tiles with varied dimensional format. The second uses dimensionally modular tiles to recreate a digital image within the contrasting grout lines.

\section{Computational Tile Patterning}

The digital workflow for creating robotically placed ceramic tile panels consists of four components. Two are used to generate parametric tile arrangements, and two are used in the robot code generation, simulation and validation. For this research all of the modules were in the form of custom components written in C\# for Grashopper $^{T M}$, the visual programming interface for Rhinoceros.

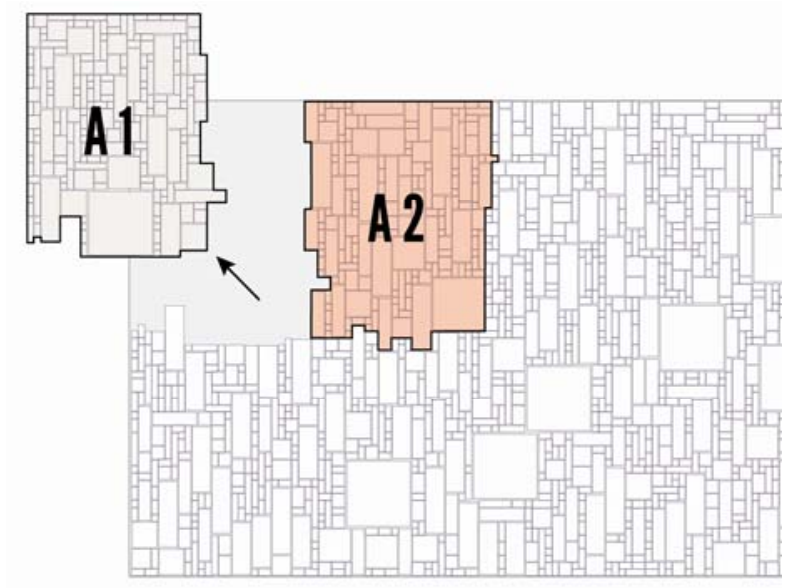

Fig 1. Generated pattern using weighted randomness.

\section{Pattern Based Digital Workflow}

A series of algorithms were developed to enable packing tiles of various formats into orthogonal patterns characterized by various degrees of regularity and randomness. Packing areas, defined by curves, and the resolution of the grid on which the pattern is based are the primary parameters of the system. Variables control the degree of randomness in the pattern and the number of tiles of each format. The pattern can accommodate any tile size, however, when using standard tile formats whose dimensions are not necessarily perfect multiples of each other, the script allows for irregular tile spacing in order to accommodate the discrepancies. Possible applications of this system include situations where there is a desire to create patterns possessing a degree of irregularity while using standard format tiles. 


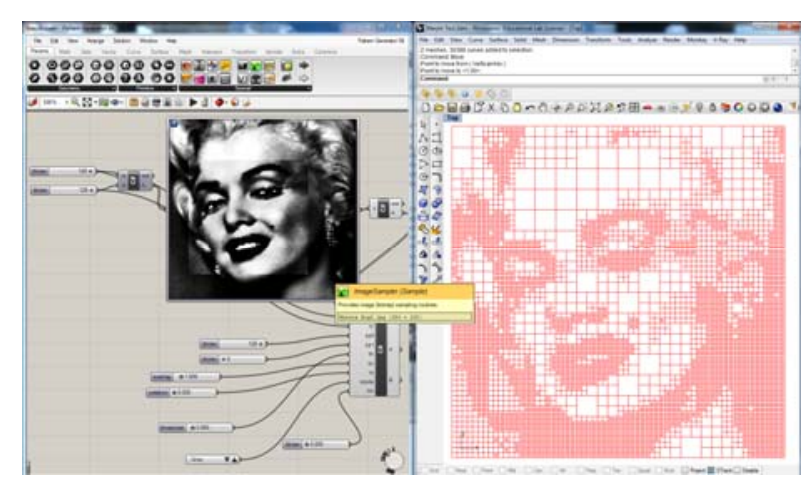

Fig 2. Image-based process converts a grayscale image into a tile pattern. The image is recreated through the density of grout lines. ${ }^{7}$

\section{Image Based Digital Workflow}

The image-based strategy uses the density of the grout lines in order to express the tone variations of the input image. As a proof of concept an iconic portrait of Marilyn Monroe was chosen to demonstrate the ability of the script to recreate something as complex, and uniquely identifiable, as the human face.

Expanding upon the pattern-based system the image-based workflow focused on maintaining equal spacing between tiles thus requiring the use of a modular tile system dimensioned on a grid. A grayscale image is used in order to drive an adaptive subdivision scheme which results in smaller tiles clustered near either darker areas or sharper edges. A grayscale translation (Fig 2) creates the most photorealistic result by converting lighter areas to larger tiles with fewer grout lines. In addition, it is possible to give the impression that tiles are following the contours in the image by increasing the grout areas and rotating the tiles so that they align with the image gradient.

\section{Integrated Programming of Robotic Placement}

The third module in the workflow converts any set of Rhino surfaces, representing tiles, into a series of movement commands for the ABB robotic arm. In Fig. 3 the lines indicate this robotic movement sequence, from a source point, to an intermediary point above the source, across the table to an intermediary point above the destination, and finally directly down into the destination. Intermediary points are necessary in the movement to avoid collision and to ensure the motion of the final placement is perpendicular to the placement surface.

The module takes as input any number of 'destination' and 'source' tile positions modeled as Rhino surfaces. The module can accommodate stacked source tiles by inputting the number and thickness of tiles at each source position. The script then automatically identifies and matches every destination tile to its corresponding source tile stack. The program keeps track of the number of tiles in each stack and adjusts the elevation of the suction gripper accordingly. Multiple stacks can also be defined. In this case the module will direct the robot to source each tile from the stack of the right type until the stack is empty, at which point an alternate stack is chosen. If no stack information is provided the script assumes the source location is 'infinite', meaning that tiles are replenished every time one is removed. The robot will continue to revisit the same source location until the process is complete. Additional work object or backer board dimensions serve as input parameters that will universally translate all of the destination tiles vertically.

The module then outputs a text file written in RAPID code, the instruction language of $A B B$ robots. This file can be loaded directly into the robot to begin the process. It is typically necessary to simulate robotic movements in the proprietary ABB software RobotStudio. In order to bypass this step and make the workflow seamless an inverse kinematic solver, previously developed by DRG contributor Panagiotis Michalatos, was used to visualize the robotic movements. This fourth module is independent and the robotic code can be generated separately if desired. However, the visualization of the robot movements within the Rhino environment greatly reduces the likelihood of error. Each file was simulated in both RobotStudio and the inverse kinematic solver to validate the tool.

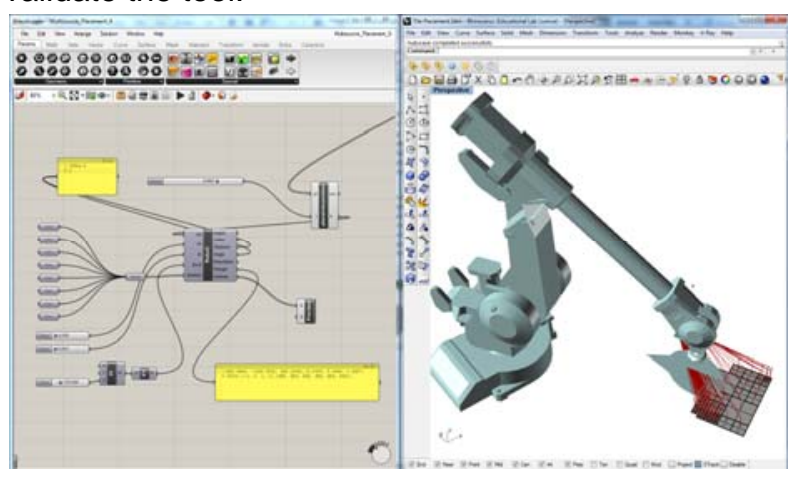

Fig 3. Simulation of movement commands for one of nine tile sections within one panel of the image based Marilyn Monroe prototype.

\section{Applied Prototype DeVelopment}

In order to better understand the parameters associated with robotic tile placement and its feasibility for the production of custom surface patterning a series of prototypes were completed using an ABB 4400 industrial robotic work cell and pneumatic suction gripper assembly.

\section{Experimentation}

Experiments were conducted involving dry placement of individual tiles at a prescribed location. The 
final positioning was evaluated within machine tolerances through a comparison of the prescribed corner locations in the digital model and the actual corner locations of each tile using custom robotic tooling. Deviation in square tile placement was acceptable when accounting for actual dimensional variation within the tiles themselves. When using non-square rectangular tiles with the added complexity of a 90degree rotation the impact of any calibration variation was exaggerated resulting in a slightly lower, but visually acceptable, accuracy.

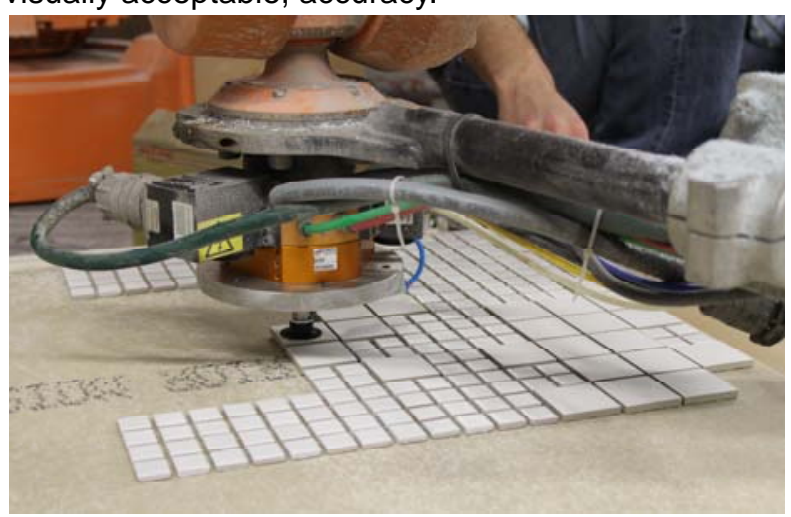

Fig 4. Dry placement of tiles during early tests.

\section{Pattern-Based Prototypes}

Two prototyping exercises were conceived that utilized the previously mentioned pattern generation techniques to enable testing of the automated robotic tile placement workflow and address the calibration concerns above.

\section{Prototype: Format Variation}

Using patterns based on tile format variation a series of $7500 \mathrm{~cm}^{2}$ compositions were created from a combination of three different sizes of square and elongated rectangular tiles arranged using a $4 \mathrm{~mm}$ grout line that was established to accept machine tolerances and dimensional inconsistency. A custom end effector was prototyped that incorporates a suction gripper assembly with a specialized housing designed to apply distributed force to the tile for 'placing' while remaining within the working range of the suction gripper for 'picking'.

During the initial series of prototypes certain physical limitations were realized. First, in a stacked tile supply the aggregate thickness variation within one stack of tiles often proved to be outside of the working tolerances of the existing custom tooling. Additionally the registration of the remaining stacked tiles was compromised as tiles were removed from the stack; as one tile was removed the tile below often shifted. These inconsistencies will be addressed during further tooling development.

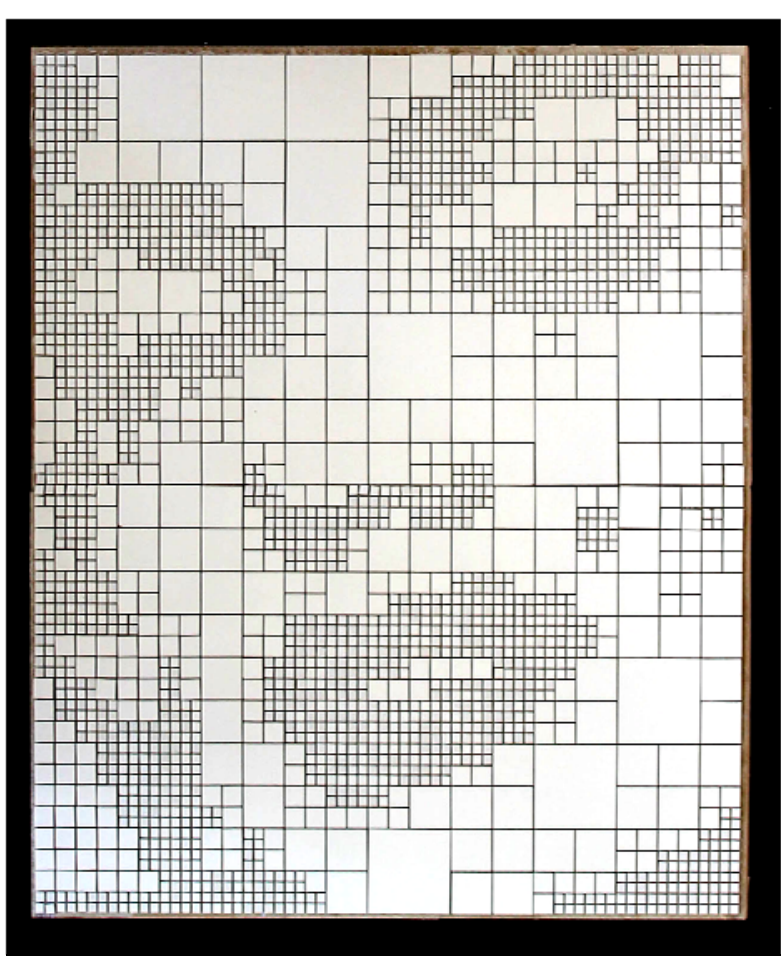

Fig 5. Completed Marilyn Monroe image-based robotically placed tile pattern prototype.

\section{Prototype: Image-Based Pattern}

For the second prototyping exercise a tiled surface was designed using the image based patterning system that was divided into two panels to test the ability of robotically placed tiles to create multiple individual modular tiled surfaces that relate to a final assembly. In this example the iconic Marilyn Monroe image required 2247 individually placed tiles of dimensionally doubling modular square tiles ranging from $19.05 \mathrm{~mm}$ to $173.4 \mathrm{~mm}$ accounting. A final assembled dimension of $1530 \mathrm{~mm} \times 1830 \mathrm{~mm}$ was constructed using two industry standard $12 \mathrm{~mm}$ cement backer boards.

Two tile adhesion strategies were tested. On one panel slightly elastic tile mastic, and on the other a double-sided flexible tile adhesive sheet, was used. To accommodate for production time in the masticbased sample, the pattern was divided into sections based on the set time of the adhesive. Periodic tiles were not included in the placement to allow for mechanical fastening during on-site installation; these voids would be tiled on site. The second panel utilized an adhesive sheet with an extended set time allowing the entire panel to be completed as one continuous process. More testing is needed to determine the best adhesive and backer board for a given scenario.

All tiles were placed robotically. The two panels were assembled and tile spacing was measured using previously described methods. Overall the grout lines remained consistent and only slight rotational 
variation was observed. The resulting installation accurately represents the prescribed digital image and the face of Marilyn Monroe is readily recognizable thus validating the image-based design-torobotic-tile-placement workflow.

\section{Expanding on the State of the ART}

The prototyped solution used affordable technology, employing an off-the-shelf factory-based industrial robot and widely available inexpensive software. Robotic tile placement requires the development of a new construction technique that allows tiles to be placed robotically in a location (factory) separate from the final installation (building or construction site) (Scenario A). Without significant advancements in on-site robotics, tiles could only be placed directly onto final and finished surfaces in the case of prefabricated construction (Scenario B). Issues for both scenarios are discussed in the following sections.

\section{Construction Scenario A: On-site Installation of Factory Produced Modules}

In this scenario tiles are robotically installed onto backer boards and then transported to the construction site for installation. The basic tile installation sequence would be as follows:

- After the tiling pattern is computationally generated the backer board subdivision is determined. Boards are labeled and an installation drawing is produced.

- Backer boards are cut using a CNC machine

- Backer boards are fixed in a jig or vacuum bed within the robotic work cell and an adhesive is applied.

- Tiles are robotically placed on the board.

- Each board is transported to a drying station while insuring limited deflection.

- The process is repeated for each board.

- Each board is labeled with a unique ID relating to the computationally generated installation drawing.

- Tiled boards are packed and shipped to site.

- An on site installer mounts the pre-tiled boards onto the rough wall or floor surface.

- Missing tiles for edges and surrounding fixtures are cut and installed by hand.

- The surface is conventionally grouted and finished.

\section{Tile Backer Board}

\section{Material and Bonding Agent}

The typical backer boards include cement backer units (also called CBU; ANSI A108.11, ANSI A118.9, ASTM C 1325), fiber cement backer board (ANSI A108.11, ASTM C1288), and glass mat waterresistant gypsum backer (ASTM C1278). ${ }^{8}$ Boards are usually $12.5 \mathrm{~mm}$ thick and come in standard formats around $0.9 \mathrm{~m} \times 1.5 \mathrm{~m}$. The choice of backer boards determines the substance needed to bond the tiles to the board. Latex/polymer modified Portland cement mortar, and epoxy are recommended options for all three boards. Dry set mortars are an option for $\mathrm{CBU}$, while organic adhesives present another option for the other two board types. High quality bonding agents are key in preventing tiles from falling off, cracking, or partially de-bonding from the backer board.

\section{Backer Board Size and Deformation}

Based on the maximum allowed U.S. lifting weight of $13.6 \mathrm{~kg}$ (30 lbs, overhead) to $18.1 \mathrm{~kg}$ (40 lbs not overhead $)^{9}$ the maximum size of a typical pre-tiled concrete backer board would be $0.46-0.65 \mathrm{~m}^{2}$ (5 to $7 \mathrm{ft}^{2}$ ) when installed by one person, and twice that amount for a two-person team. Size limitations will also depend on the panel flex and the risk of tile breakage. According to a report by the Ceramic Tile Institute of America, Inc., tile floors installed over wood joist systems can deflect up to $11.4 \mathrm{~mm}(0.45$ in.) over a span of $4.06 \mathrm{~m}$ (13.33 ft) without risk of cracked floor tiles. ${ }^{4}$ Extrapolating that data, and assuming a backer board segment $1.20 \mathrm{~m}$ (4 ft) long, the sheet could deflect by $3.3 \mathrm{~mm}$ (0.13 in.) without risk of tile cracking. The maximum permissible deformation depends to a large degree on the tile format, the material used as the backer board, and on how boards are stored, transported, and handled on site. A computational deflection study (assume concrete backer board with $\mathrm{f}_{\mathrm{c}}{ }_{\mathrm{c}}=20,684 \mathrm{kPa}$ or $3,000 \mathrm{psi}$ and $9 \mathrm{~mm}$ or $3 / 8 \mathrm{in}$. tile surface with tiles measuring 50 by $75 \mathrm{~mm}$, placed with 4 in grout joints) of a board measuring $1.2 \mathrm{~m} \times 0.45 \mathrm{~cm}(4 \times 1.5 \mathrm{ft})$, carried by two people in the long direction showed $9 \mathrm{~mm}$ (0.35 in.) of deflection from dead load.

\section{Backer Board Cutting}

Water-jet cutting would be a cost-effective method of trimming backer boards to a size that matches the tile pattern. Using an automated tool changer waterjet heads could be mounted on the same robot used for tile placement, as is currently done at Harvard's robotic work cell.

\section{Robotic Tile Placement Technique}

When installing tiles by hand it is recommended to beat them in gently using a beating block or a rubber mallet, ensuring the back surface of the tile fully adheres to the bonding agent. Further research is needed to determine the appropriate robotic techniques for achieving the same effect. Force sensors at the robotic end effectors can be configured to apply tiles with even pressure, potentially improving the consistency of installation compared to manual installation. 


\section{Backer Board Mounting}

Backer boards with pre-installed tiles need to be installed on site using commonly available techniques. Adhesives, mechanical connectors, and combinations thereof are possible. Backer boards are normally screwed directly into the studs (wood or metal stud construction) or bonded with thin-set mortar onto masonry or concrete walls. Mechanically connecting into the studs is not feasible for pre-tiled backer boards because tile patterns and stud patterns will rarely align. Backer boards should thus be adhered to another surface - a layer of sheet wood or concrete board in the case of stud construction, or directly to the wall surface in the case of masonry construction.

In manual tile installation a gap of $2-3 \mathrm{~mm}$ is recommended between adjacent backer boards, normally to be filled with mortar and taped to prevent water penetration. In the case of the pre-tiled system moisture barriers would be applied onto the surface behind the pre-tiled backer boards. Backer boards can be cut such that a slight gap, the size of the grout joint, remains between boards in order to allow for adjustments in response to normal construction tolerances. Total system thickness for stud construction will increase by approximately $12.5 \mathrm{~mm}$ because pre-tiled backer boards require an initial board to be installed onto the studs.

\section{Scenario B : Direct Robotic Placement during Pre-Fabrication}

In pre-fabricated construction tiles can be installed directly onto a surface prepared by other trades. The following process would apply:

- The tile surface is prepared in accordance with the standards of the adhesive.

- Adhesive is robotically applied to the board surface.

- Tiles are robotically placed onto the surface.

- Once the tile bond dries the surface is conventionally grouted and cleaned by hand.

\section{Edge and Adjustment Tiles}

The realities of construction will usually require a certain number or tiles to be cut to fit around fixtures or to make up the distance between the regular tile pattern and the edge of the surface. For Scenario A the cut tiles will be mounted on-site prior to grouting, for Scenario B the cut tiles can be installed directly in the factory. In the future it may be possible to pre-cut most odd shaped tiles based on as-built laser scans of the surface to be tiled.

\section{FinANCIAL FeAsibiLITY StUdy}

Using the prototypical panel described the approximate costs of robotic tile placement based on current
U.S. industry standard labor and robotic machine rates are as follows:

- $\$ 86-\$ 110 / \mathrm{m}^{2}$ for standard tile patterns*

- $\$ 270-\$ 430 / \mathrm{m}^{2}$ for mosaic tiles pre-mounted on 30 × $30 \mathrm{~cm}$ sheets*

*Average manual tile installation cost including edge trimming and grouting. Tile installation costs can vary based on location; New York City for example can be more than twice as expensive, while rural areas will have significantly lower costs. ${ }^{5,10}$

Comparatively robotic machine time including 1 operator is $\$ 120$ / hr including all overhead. The prototype measures $2.78 \mathrm{~m}^{2}$, and features a total of 2,247 tiles of four different sizes. The study assumed several robotic placement rates determined in both computation simulations as well as derived from the experiments at the GSD. These placement rates were 7,10 , and 12 seconds on average per tile. Setup time for the panel was estimated at one hour in a professional production context. On-site installation of the pre-tiled surface was assumed to take 2 man-hours, and grouting was estimated at 3 hours. The comparative calculation assumed the above mentioned manual tile installation costs. The cost rates quoted assume net-mounted mosaic tiles in 30 $x 30 \mathrm{~cm}$ sheets. Rates for complex patterns such as the one enabled by robotic tile placement are not available, but can be estimated to be much higher.

The study shows that robotic tile placement could be competitive as far as installation costs are concerned. Within the range of placement parameters (both robotic placement rate and manual installation costs) the cost for an installed square meter of tiled surface is in the range of \$380-540 for both robotic and manual methods. Given the added value of customizing the tile pattern for robotic placement it seems clear that the technique would be feasible in practice from a cost point of view.
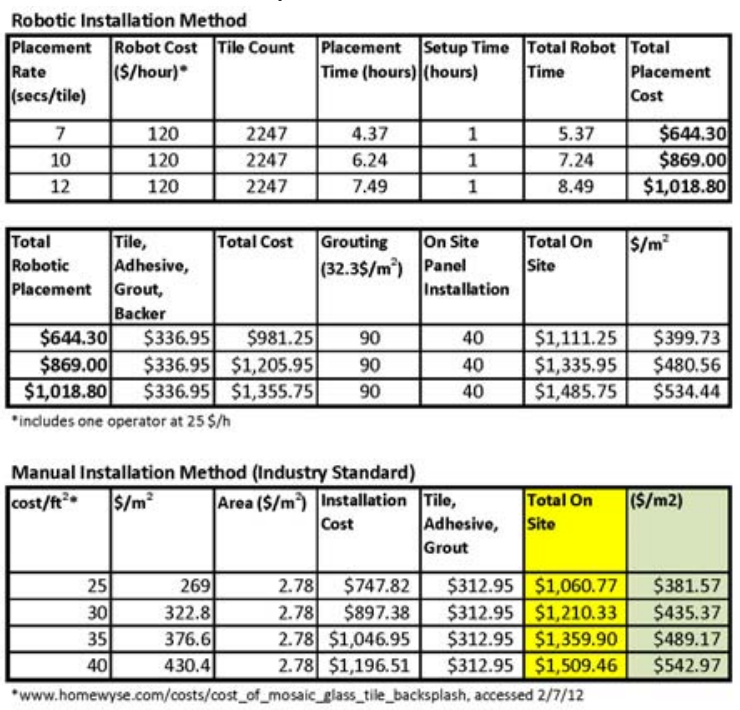

Fig. 6 Financial feasibility study results 


\section{Estimated Capital Costs}

Investment costs for a robotic tile placement cell designed to run constantly for 5 years on average are estimated to be in the range of $\$ 100,000$ to $\$ 200,000$. The software needed to run the cell is extremely low-cost, the commercial retail value of the package at present being $\$ 1,000 /$ seat without the additional digital tools developed by the GSD research team.

\section{FutuRE DEVELOPMENTS AND OUTLOOK}

Format customization as an added opportunity remains to be explored as a next step. Format customization could be seen in the following contexts:

- Tile formats are customized on certain production lines allowing a wider range of placement patterns.

- Customization may take into account as-built dimensions of walls and floor surfaces, creating customized tiles that eliminate or drastically reduce on-site cutting waste. Laser scanning as-built surfaces would allow the integration of precise geometry data into the pattern algorithm.

- Tile patterns using leftover tiles, creating value by using unwanted products. Cutting leftover tiles is also possible to avoid format limitations.

- stochastically generate tile patterns based on broken tile (tile trash) from factories or sites where tiles are installed in conventional ways.

Next steps in the technology development include improving the user interface of the computational pattern generator, as well as a possible web-based interface. Refined custom robotic tooling could be developed using force-sensing and other technologies as agents of quality control.

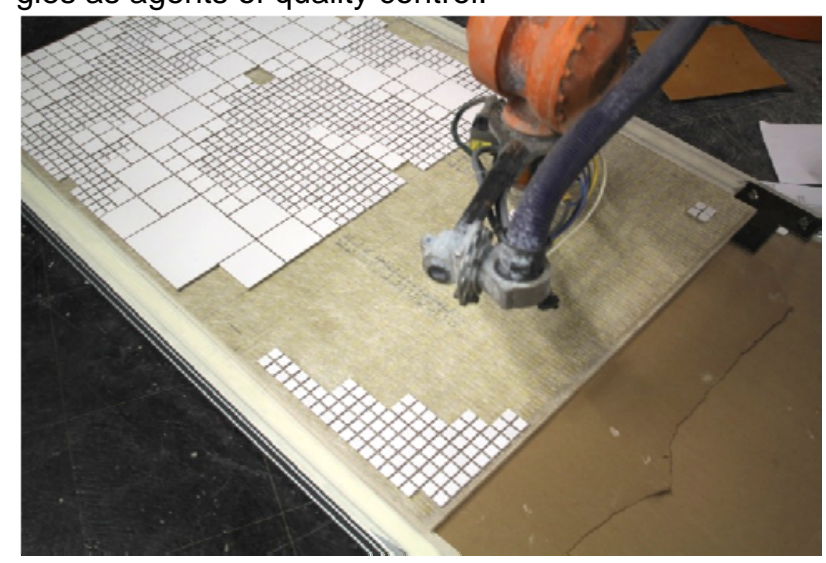

Fig. 7 One panel of the image prototype

\section{Conclusion}

The need for tile customization is growing, as the success of ink-jet printing on ceramic tiles in Spain is demonstrating. The potential market would likely be medium to high end consumer and commercial, but also infrastructure and transport (e.g. subway stations etc.) applications.
The research studied the potential for robotic placement of industry-standard pressed tiles using a computational workflow that allows complex patterns to be created based on a variety of mathematical algorithms and image based methods. The generation of machine code, process simulation, and ultimately robotic tile placement can be automated using the proposed integrated design-to-robotic-fabrication workflow. Several initial tests were conducted to determine the feasibility of the technology.

A final prototype measuring 1.8 by 1.5 meters was produced as a proof of concept, demonstrating that the novel technology can indeed be employed to produce complex tile patterns impossible to install economically by hand. An initial cost comparison shows that installation costs for both robotic and manual placements are similar, but robotic methods can produce highly varied, custom patterns.

\section{ACKNOWLEDGMENTS}

Research funded by a grant from ASCER Tile of Spain.Amanda Lee, Research Associate, Harvard GSD DRG

\section{References}

1. Bechthold, M. et. al., "Integrated Environmental Design and Robotic Fabrication Workflow for Ceramic Shading Systems", Proceedings of 28th International Symposium on Automation and Robotics in Construction (ISARC2011), Seoul, 2011.

2. Lichtenberg, J., "The Development of A Robot for Paving Floors With Ceramic Tiles", Proceedings of the 20th International Association for Automation and Robotics in Construction, Eindhoven, Holland, pp 85-88, 2003.

3. Oral, A., and Erzincanli, F., "Computer assisted robotic tiling of mosaics", Robotica, Vol. 22, pp. 235239, 2004.

4. deBear, D., "Ceramic tile over Wood Sub-Floors Regarding Deflection", CTIOA (Ceramic Tile Institute of America Inc.), Field Report 2001-11-19, Culver City, 2001.

5. Acworth, T., interview by Martin Bechthold, Boston, MA, June 1, 2011.

6. Top Hat Tile Customized Mosaics, "Architectural mosaics", accessed July 15, 2011, http://www.tophattile.com.

7. Monroe, M., "The Asphalt Jungle", 1950, publicity still, IMDB http://www.imdb.com/media/rm4267350016/tt00422 08 , accessed February $2012^{\star}$ Assumed to be public domain

8. ANSI: American National Standards Institute, ASTM: American Society for Testing and Materials.

9. Waters, T.R., Puts-Anderson, V., Garg, A., "Applications Manual for the Revised NIOSH Lifting Equa- 
tion", US Department of Health and Human Services, 1994.

10. Homewyse, "Cost of Ceramic Tile Flooring", last modified March 2012,

http://www.homewyse.com/costs. 